\title{
The role of the deubiquitinating enzyme DUB3/USP17 in cancer: a narrative review
}

\author{
Guang-Fei Yang ${ }^{1}$, Xin Zhang ${ }^{2}$, Yi-Ge Su ${ }^{3}$, Ren Zhao ${ }^{4,5}$ and Yan-Yang Wang ${ }^{4,5^{*}}$ (1)
}

\begin{abstract}
The balance between ubiquitination and deubiquitination is critical for the degradation, transport, localization, and activity of proteins. Deubiquitinating enzymes (DUBs) greatly contribute to the balance of ubiquitination and deubiquitination, and they have been widely studied due to their fundamental role in cancer. DUB3/ubiquitin-specific protease 17 (USP17) is a type of DUB that has attracted much attention in cancer research. In this review, we summarize the biological functions and regulatory mechanisms of USP17 in central nervous system, head and neck, thoracic, breast, gastrointestinal, genitourinary, and gynecologic cancers as well as bone and soft tissue sarcomas, and we provide new insights into how USP17 can be used in the management of cancer.
\end{abstract}

Keywords: DUB3/USP17, Cancer, Ubiquitination, Deubiquitinating enzymes, Signaling pathways

\section{Introduction}

Protein is the basic unit that regulates many cellular processes in eukaryotic cells. Ubiquitination is the second most common posttranslational modification (PTM), and it modifies the activity, localization, interaction, and stability of proteins [1]. Furthermore, ubiquitination also affects many other cellular and biological processes, such as cell cycle control, DNA repair, transcriptional regulation, immune response, and apoptosis [2]. Therefore, abnormal ubiquitination may lead to various diseases, including cancer $[3,4]$.

The ubiquitination process is achieved by the following three types of enzymes: ubiquitin-activating enzyme (E1), ubiquitin-conjugating enzyme (E2), and ubiquitin ligase (E3) [5]. The ubiquitination process can also be reversed by deubiquitinating enzymes (DUBs) [6], which affect various signaling pathways by removing ubiquitin from substrates [7]. Ubiquitin-specific proteases (USPs) account for the largest proportion of DUBs in the human genome with nearly 70 members [8]. In recent years, the

*Correspondence: fdwyy1981@hotmail.com; wangyy@nxmu.edu.cn

${ }^{4}$ Dept. of Radiation Oncology, General Hospital of Ningxia Medical University, Yinchuan 750004, Ningxia, China

Full list of author information is available at the end of the article oncogenic or tumor suppressive function of USPs has been assessed in many studies [9], and all studies have demonstrated the potential role of USPs against cancer.

USP17, also referred to as DUB3, is regulated by interleukin-4 (IL-4) and IL-6 cytokines [10]. The abnormal expression of USP17 is related to inflammation, cell motility, the development of $\mathrm{T}$ helper 17 (Th17) cells, and carcinogenesis [11]. Several studies have found that USP17 plays a critical role in the carcinogenesis and progression of different types of cancer, but an in-depth review on the role of USP17 in cancers is lacking. Therefore, this review summarized the biological functions and regulatory mechanisms of USP17 in various types of cancer to provide new possibilities for the management of cancers.

\section{Ubiquitination process}

Ubiquitination refers to the process of covalent conjugation of ubiquitin, which is a protein composed of 76 amino acids that attach to a lysine residue on substrate proteins. The process requires the following three types of enzymes: E1, E2, and E3. In humans, there are 2 E1 enzymes, approximately 50 E2 enzymes, and approximately 600 E3 ubiquitin ligases [12].

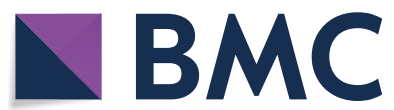

(c) The Author(s) 2021. Open Access This article is licensed under a Creative Commons Attribution 4.0 International License, which permits use, sharing, adaptation, distribution and reproduction in any medium or format, as long as you give appropriate credit to the original author(s) and the source, provide a link to the Creative Commons licence, and indicate if changes were made. The images or other third party material in this article are included in the article's Creative Commons licence, unless indicated otherwise in a credit line to the material. If material is not included in the article's Creative Commons licence and your intended use is not permitted by statutory regulation or exceeds the permitted use, you will need to obtain permission directly from the copyright holder. To view a copy of this licence, visit http://creativecommons.org/licenses/by/4.0/. The Creative Commons Public Domain Dedication waiver (http://creativecommons.org/publicdomain/zero/1.0/) applies to the data made available in this article, unless otherwise stated in a credit line to the data. 
In the first step, E1 hydrolyzes adenosine triphosphate (ATP) and catalyzes the C-terminal acyl adenylation of ubiquitin. Ubiquitin is then transferred to the cysteine residue of the active site of $\mathrm{E} 1$, which is accompanied by the adenylation of the second ubiquitin. The adenylated ubiquitin is then transferred to the cysteine residue in the ubiquitin conjugation domain of E2. The final step of conjugating ubiquitin to the target is mediated by E3 ubiquitin ligases. E3 is responsible for substrate identification and transfer, and it plays a key role in determining the specific type of ubiquitinated substrate $[13,14]$.

According to the different catalytic structures, E3 enzymes can be divided into three families as follows: really interesting new gene (RING), homologous to the E6-AP carboxyl terminus (HECT), and the ring between ring fingers (RBR) family [15]. RING is the largest family of E3 ligases that help transfer ubiquitin connected to E2 directly to the substrate without forming a thioester bond with ubiquitin $[16,17]$. The amount of HECT E3 is less than that of RING E3. HECT E3 forms catalytic cysteinedependent intermediates with ubiquitin linked to E2, which are then transferred to the target protein [18]. RBR is a special type of E3 ligase, and its activation mechanism is different from that of RING and HECT. RBR has two RING domains (RING1 and RING2) and a domain between the RING domains (IBR). The RING1 domain initially recognizes the ubiquitin attached to E2, and the RING2 domain then provides a cysteine residue to the active site of E2, which forms a thioester-linked E2-ubiquitin intermediate $[19,20]$.

Ubiquitination can be divided into polyubiquitination and monoubiquitination based on the number of ubiquitins attached to proteins [21]. Different types of ubiquitination modifications are related to different physiological functions in cells. Usually, the polyubiquitination process is involved in both protein degradation and signal transduction. For example, Lys-48(K48)-branched polyubiquitination regulates protein stability through $26 \mathrm{~S}$ proteasome-mediated degradation. The K63-linked polyubiquitination chain executes a nonproteasome process. However, monoubiquitination is involved in the regulation of diverse cellular processes, such as DNA repair, signal transduction, receptor endocytosis, and stress response [22, 23].

The ubiquitination process can be reversed by DUBs, which belong to the family of cysteine proteases. DUBs cleave the isopeptide bond or the peptide bond with high specificity, and they are responsible for removing ubiquitin from the substrate and maintaining its stabilization [24]. DUBs can be divided into the following six families according to sequence and structure: ubiquitinspecific proteases (USPs), ubiquitin $\mathrm{COOH}$ terminal hydrolases (UCHs), ovarian tumor proteases (OTUs),
Machado-Joseph (Josephin) domain proteases (MJDs), JAMM/MPN domain-associated metallopeptidases (JAMMs), and monocyte chemotactic protein-induced proteins (MCPIPs) [8, 25-27]. These enzymes directly bind to different types of ubiquitin chains and remove ubiquitin chains from the substrates. Engineered deubiquitination synthesis shows that OTUs specifically remove K29-linked ubiquitin chains from the substrate, while JAMMs are often unique to K63-linked ubiquitin chains. Among the DUB subfamilies, the USPs are highly diversified and comprise more than 50 members, forming the largest subfamily of DUBs. USPs undergo mutations in multiple biological processes and are frequently altered in cancers [28, 29] (Fig. 1).

\section{Structure of DUB3/USP17}

The DUB3/USP17 genes are mainly located on chromosome 4 (4p16.1) and partly on chromosome 8 (8p23.1). According to their sequence homology, human USP17 is divided into 13 subfamily members (USP17A-N) [30], which are encoded by the human mega satellite tandem repeat sequence (RS447) and have unique expression patterns [31]. USP17 contains a USP domain and two hyaluronan (and RNA) binding motifs (HABMs) (Fig. 2). The USP domain is located near the $\mathrm{N}$-terminus, and HABMs are located at amino acids 401-409 and 445-453 near the C-terminus. Each USP domain contains a catalytic component [Cys, Asp (I), His, and Asp/Asn (II)] and is responsible for deubiquitination activity [32]. HABMs exist in the C-terminus of USP17 subfamily members, except in USP17N. HABMs participate in the interaction between USP17 and hyaluronic acid (HA), which inhibits cell proliferation and anchorage-independent tumor growth [33]. In addition, the C-terminal domain also has a role in the catalytic process of USP17, and its deletion significantly reduces the activity of USP17.

\section{Role of DUB3/USP17 in cancer}

\section{Central nervous system cancer}

Glioma is one of the most common tumors in the central nervous system [34]. The expression level of USP17 in glioma tissues is lower than that in normal brain tissues. Forced expression of USP17 inhibits the carcinogenesis and proliferation ability of glioma cells by reducing the protein levels of Ras and Myc. An in vivo orthotopic model has also shown that overexpression of USP17 inhibits glioma progression. A cohort study of 104 patients with glioma has revealed that the expression level of USP17 is negatively correlated with WHO grade [35]. Another study [36] has reported that DUB3 and WEE1, as downstream regulators of the phosphatase and tensin homolog (PTEN)/protein kinase B (Akt) pathway, negatively regulate cell cycle progression and 

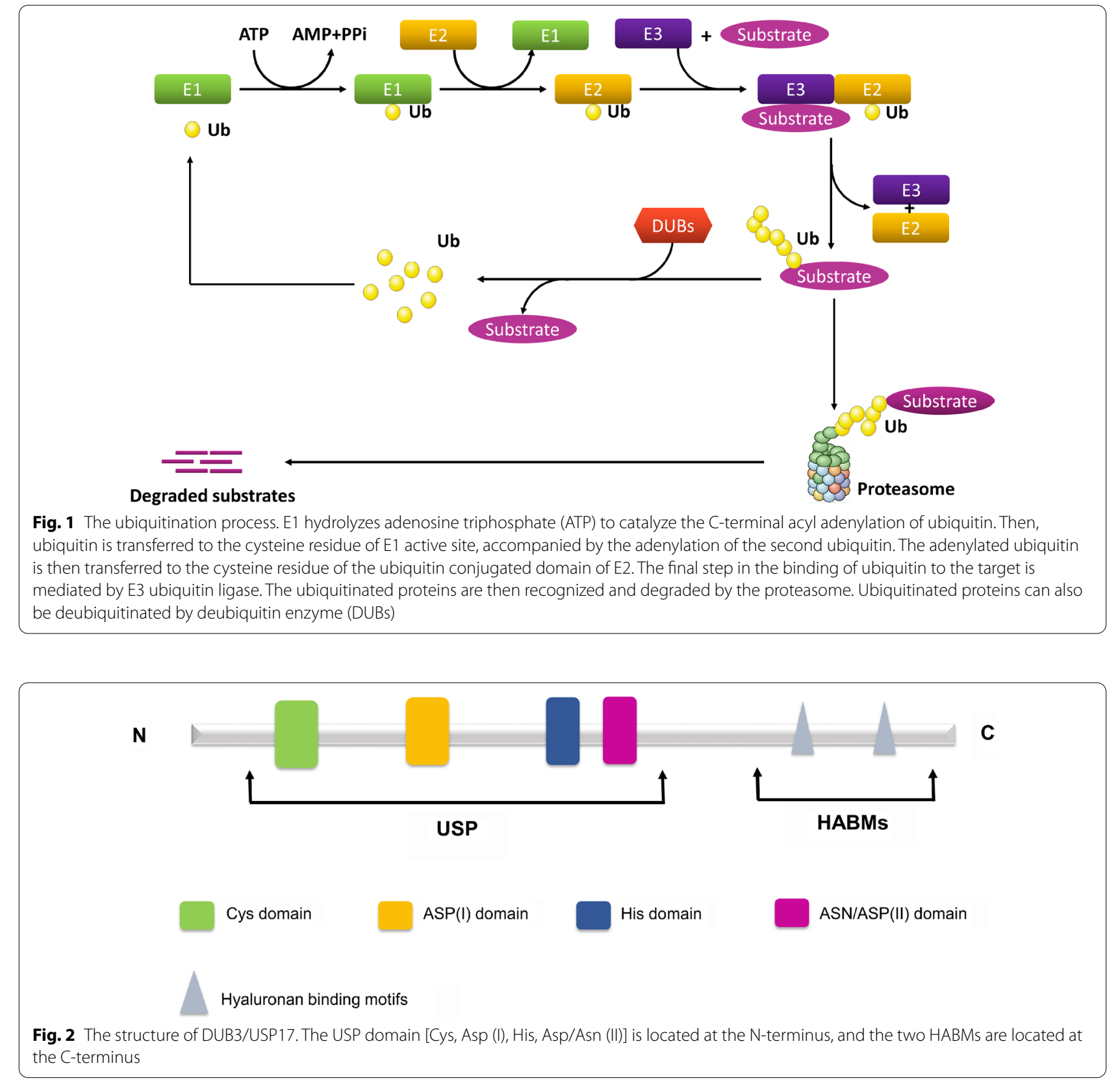

proliferation of glioma cells through cell division cycle 25A (CDC25A). These findings suggest that USP17 exerts a tumor suppressor role in glioma.

\section{Head and neck cancer}

DUB3 is overexpressed in oral squamous cell carcinoma (OSCC) tissues and cell lines, and it has a negative effect on the survival of patients with OSCC. Mechanistically, DUB3 promotes the proliferation of OSCC cells and inhibits apoptosis by triggering the expression of enhancer of zeste homolog-2 (EZH2) by suppressing ubiquitin-mediated bromodomain-containing protein 4 (BRD4) degradation [37].

The tumor suppressor role of the long noncoding RNA, LINC02487, has been demonstrated in OSCC. Silencing LINC02487 leads to the activation of migration and mesenchymal characteristics of OSCC. However, these effects are rescued by downregulating USP17. LINC02487 directly binds to USP17 and negatively regulates USP17. As a downstream molecule of LINC02487, USP17 further exerts its role in epithelial mesenchymal transition (EMT) through posttranslational deubiquitination and 
stabilization of Snail1 [38]. These studies suggest that DUB3 may be an effective anticancer target for OSCC.

\section{Thoracic cancer}

Many studies have investigated the expression level of USP17 in lung cancer tissues and its clinical significance. McFarlane et al. [39] reported that USP17 is overexpressed in both lung squamous cell carcinoma and adenocarcinoma. However, the expression level of USP17 in squamous cell carcinoma is higher than that in adenocarcinoma. Moreover, the relapse-free survival rate of patients with USP17-positive cancer is significantly lower than that of USP17-negative cancer [39].

Increasing evidence shows that DUBs are involved in the regulation of the cell cycle [40]. It has been reported that deletion of DUB3 blocks the cell cycle of non-small cell lung cancer (NSCLC) cells from G1 to S phase. Mechanistically, DUB3 drives cell cycle progression and promotes lung cancer cell proliferation by deubiquitinating and stabilizing cyclin A [41].

USP17 expression is induced by epidermal growth factor (EGF) stimulation, which is necessary for EGFR endocytosis [42]. Deletion of USP17 blocks the internalization of transferrin and its receptor (TfR). Because TfR is the archetypal substrate for clathrin-mediated endocytosis (CME), silencing USP17 impedes the localization of CME machinery components to the plasma membrane. The impact of USP17 depletion on growth, EGFR endocytosis, and signal transduction has been evaluated in NSCLC cells. For NSCLC cells carrying EGFR activation mutations, deletion of USP17 inhibits the growth of these cells and triggers apoptosis. In addition, deletion of USP17 blocks CME in these cells, but it has no effect on the endocytosis of EGFR mutants and downstream signal transduction. For EGFR wild-type NSCLC cells, suppression of USP17 enhances the sensitivity of the EGFR tyrosine kinase inhibitor (TKI), gefitinib [43]. These data indicate that targeting USP17 is an interesting complement to EGFR TKIs to prevent drug resistance or an alternative after drug resistance is established.

Macrophages are involved in the interaction between inflammation and lung cancer [44]. A connection among USP17 expression, macrophage accumulation, and inflammation has been reported in lung cancer [45]. USP17 is related to the expression level of inflammatory mediators, macrophage markers, and poor prognosis of lung cancer. Tumor necrosis factor receptor-associated factors (TRAFs) are important regulators of inflammatory signaling pathways. The binding motifs of TRAF2 and TRAF3 have been identified in USP17. USP17 removes the K63-linked ubiquitination of TRAF2 and TRAF3, which disrupts the TRAF2/TRAF3 complex. Thus, the target proteins of the TRAF2/TRAF3 complex, namely, NF-kappaB-inducing kinase (NIK), c-Rel, and interferon regulatory factor 5 (IRF5), are activated, which upregulates inflammation-related genes and stemnessrelated genes in cancer cells [45]. These findings suggest that USP17 drives positive feedback of macrophages and cancer cells to enhance the inflammation and stemness of cancer cells as well as to promote the progression of lung cancer.

Hyaluronan is a type of glycosaminoglycan in the extracellular matrix, and its level is regulated by both hyaluronan-synthesizing enzymes and -degrading enzymes. Due to the uncontrolled expression of the hyaluronan synthase 2 (HAS2) gene and/or changes in HAS2 activity, hyaluronan can be deposited in rapidly remodeled tissues [46]. Hyaluronan synthesized by abnormal HAS2 is closely related to the progression of solid tumors. Compared to normal tissues, the expression level of hyaluronan in lung cancer patients is significantly upregulated, and the elevated level of hyaluronan is regulated by USP17 through deconjugating polyubiquitin chains from HAS2 [47].

Additionally, Zhang et al. demonstrated that suppression of USP17 inhibits NSCLC tumorigenesis and growth by targeting matrix metallopeptidase 3 (MMP3) and MMP9 [48]. Zhang et al. [49] demonstrated that the viability of USP17-overexpressing cells treated with cisplatin is significantly higher than that of control cells. The activation of phosphoinositide-3-kinase (PI3K)/Akt signaling may be one of the contributors to USP17-mediated cisplatin resistance in NSCLC cells. Based on these results, USP17 may be an attractive target for NSCLC management.

\section{Breast cancer}

Lin et al. [50] showed that the expression of USP17 is downregulated in breast cancer tissues and breast cancer cell lines, and they reported that USP17 overexpression significantly inhibits the growth of breast cancer in vitro and in vivo. Further studies have demonstrated that the anticancer function of USP17 is related to asparaginyl endopeptidase (AEP). The ubiquitination of AEP is regulated by TRAF6, which is an E3 ligase that mediates the binding of the K63-linked polyubiquitin chain to proteins. However, the ubiquitination of AEP is reversed by USP17, which subsequently forms a complex with heat shock protein $90 \alpha$ (HSP90 $\alpha$ ), thus promoting the intracellular stability and secretion of pro-AEP. Blocking the interaction between pro-AEP and TRAF6 or inhibiting HSP90 $\alpha$ reduces the secretion of pro-AEP, thus preventing breast cancer metastasis. In addition, USP17-mediated downregulation of AEP protein levels inactivates Erk signaling and inhibits the tumorigenesis and growth of breast cancer [51]. 
SET domain-containing protein 8 (SET8) is an enzyme that catalyzes the monomethylation of H4K20 (H4K20me1) and participates in tumorigenesis [52]. USP17 is the deubiquitinase of SET8, which interacts with SET8 to remove polyubiquitin chains, thereby stabilizing SET8. Knockdown of USP17 leads to a decrease in SET8 protein levels, which subsequently induces p21 by suppressing H4K20me1 on p21. The induction of $\mathrm{p} 21$ then triggers cell senescence and prevents the proliferation of breast cancer cells [53].

Recent research has suggested that DUB3 is involved in the metastasis of triple-negative breast cancer (TNBC). In TNBC, cyclin-dependent kinases 4 and 6 (CDK4/6) activate DUB3 by phosphorylating the Ser41 site of DUB3. Activated DUB3 deubiquitinates and stabilizes Snail1, which is an important factor in promoting EMT and breast cancer metastasis. Therefore, CDK4/6 inhibitors induce DUB3 inactivation, promote Snail1 protein instability, and reduce cell migration, thus inhibiting metastasis in xenograft models of breast cancer [54]. The significance of the CDK4/6-DUB3 axis in regulating breast cancer metastasis has been confirmed by another study [55], demonstrating that the inhibitor of DUB3, WP1130, suppresses DUB3-mediated Snail1 stabilization and that IL-6 stabilizes Snail1 by activating DUB3. In addition to Snail, Slug and Twist are also regulated by the IL-6-DUB3 axis [56]. In basal-like breast cancer (BLBC) cells, IL-6 promotes metastasis by activating DUB3. Activated DUB3 further interacts with Slug and Twist to prevent the degradation of Slug and Twist by deubiquitination, thus promoting the migration, invasion, and cancer stem cell-like characteristics of BLBC cells. Therefore, DUB3 inhibitors significantly inhibit not only the upregulation of Slug and Twist induced by IL- 6 in BLBC cells but also the invasion induced by IL- 6 . These data reveal the role of the IL-6-DUB3 signaling axis in the regulation of EMT progression, suggesting the potential of DUB3 as an anticancer drug target.

CDC25A is the key molecule that promotes oncogenic transformation [57]. It has been reported that DUB3 removes the polyubiquitin modification of $\mathrm{CDC} 25 \mathrm{~A}$, which prevents its degradation, thus amplifying the oncogenic role of CDC25A in breast cancer. Knockdown of the DUB3 gene leads to cell cycle arrest in G1/S and G2/M phases. In contrast, overexpression of DUB3 promotes cell accumulation in the $\mathrm{S}$ and $\mathrm{G} 2$ phases, which is crucial for the oncogenic function of CDC25A [58]. In addition, the impaired activation of GTPases in USP17depleted cells causes the accumulation of the cyclindependent kinase inhibitors (CDKIs) p21 ${ }^{\text {cip } 1}$ and p27 $27^{\mathrm{kip} 1}$ and $\mathrm{G} 1$ phase arrest [59].

Geminin is an inhibitor of chromatin licensing and DNA replication factor 1 (Cdt1) [60], and DUB3 regulates
Geminin through deubiquitination. DUB3 overexpression increases the level of Geminin and is associated with genomic instability, DNA replication change, aneuploidy, and cancer progression [61]. These data confirm that DUB3 regulates DNA replication by controlling Geminin levels, thereby suggesting a new role of DUB3 in breast cancer progression.

In breast cancer, the combined use of bromodomain and extraterminal domain (BET) inhibitors with histone deacetylase (HDAC) inhibitors upregulates the expression level of USP17. The increased expression of USP17 leads to a reduction in the Ras/mitogen-activated protein kinase (MAPK) signaling pathway and cell viability, while siRNA-mediated USP17 silencing significantly reverses the cytotoxicity of the combined treatment of BET and HDAC inhibitors [62].

Pristimerin (20 $\alpha$-3-hydroxy-2-oxo-24-nor-friedela1-10,3,5,7-tetraen-carboxylic acid-29-methyl ester) is a natural quinone methide triterpenoid isolated from Celastraceae and Hippocrateaceae. Studies have shown that pristimerin has insecticidal, anti-inflammatory, antiangiogenic, antiprotozoal, and anticancer effects [63]. A recent study has found that pristimerin has an anticancer effect on breast cancer [64]. Pristimerin inhibits breast cancer progression by upregulating miR-542-5p. MiR542-5p binds to Argonaute 2 (AGO2) and inhibits the expression of DUB3 in breast cancer cells. These findings increase the understanding of the upstream regulator of DUB3.

\section{Gastrointestinal cancer}

Zinc finger E-box combined with homeobox 1 (ZEB1) is an important inducer of chemotherapy and radiotherapy resistance in cancers [65]. USP17 has been identified as the downstream regulator of ZEB1 in colorectal cancer cells [66]. H2A histone family member X (H2AX) is required for the recruitment of proteins involved in DNA repair [67]. As a deubiquitylating enzyme, USP17 inhibits the DNA damage-induced ubiquitination of $\mathrm{H} 2 \mathrm{~A}$ histone family member X (H2AX) [68], which may contribute to ZEB1-mediated chemotherapy resistance [66]. In addition, by directly controlling the level of H2AX deubiquitination, USP17/DUB3 regulates recruitment of the DNA repair factors p53-binding protein 1 (53BP1) and breast cancer susceptibility gene 1 (BRCA1), in DNA damage sites to ensure a correct DNA damage response (DDR) [68]. USP17 is also involved in the repair of DNA damage by mediating defective chorion-1 (DEC1), a molecule that plays a key role in DNA damage repair. To cope with DNA damage caused by genotoxic stress, USP17 activates and stabilizes DEC1 through deubiquitination. Subsequently, DEC1 is degraded by $\mathrm{SCF}^{\beta \operatorname{TrCP}}$ ubiquitin ligase and CK1 $\alpha$ on the proteasome, which is necessary 
for effective recovery from the G2 DNA damage checkpoint [69].

Finally, it has been reported that DUB3 leads to chemotherapy resistance through Nrf2 deubiquitination and stabilization [70]. The interaction between DUB3 and $\mathrm{Nrf2}$ removes the K48-linked polyubiquitination of $\mathrm{Nrf2}$, which ultimately inhibits the degradation of Nrf2 and allows formation of a functional complex of Nrf2 and Keap1, resulting in DUB3-induced chemotherapy resistance.

\section{Genitourinary cancer}

Prostate cancer is one of the most common male cancers in the world [71]. USP17 is highly expressed in prostate cancer tissues and cell lines. Prostate cancer patients with low expression levels of USP17 have a better overall survival rate. Inhibition of USP17 expression significantly induces apoptosis and downregulates the proliferation, migration, and invasion of prostate cancer cells. The anticancer effect of USP17 has been verified in a subcutaneous mouse model. Mechanistically, inhibition of USP17 blocks NF-KB signal transduction by promoting reactive oxygen species (ROS) production [72].

DUB3 binds to BRD4 to promote its deubiquitination and stabilization in prostate cancer. The sensitivity of prostate cancer cells to the BET inhibitor, JQ1, depends on the deubiquitinating effect of DUB3 on BRD4. Therefore, DUB3 inhibitors may promote the degradation of BRD4 and reverse the drug resistance effect of JQ1 in prostate cancer. DUB3 itself is transcriptionally repressed by the nuclear receptor corepressor 2 (NCOR2)-HDAC10 complex. NCOR2 deletion leading to activation of DUB3 and BRD4 protein is often detected in castrated prostate cancer patients. These findings suggest that DUB3 leads to drug resistance to $\mathrm{BET}$ inhibitors by stabilizing the BRD4 protein, suggesting that DUB3 is a feasible therapeutic target for overcoming the drug resistance of BET inhibitors in prostate cancer [73].

\section{Gynecologic cancer}

It has been demonstrated that overexpression of USP17 significantly induces apoptosis and inhibits cell proliferation in cervical adenocarcinoma cells. Further study has revealed that the inhibitory effect of USP17 depends on suppressor of defective silencing 3 (SDS3)-related HDAC activity. The C-terminus of USP17 binds to the N-terminus of SDS3 and colocalizes in the nucleus, specifically deubiquitinating the K63-linked polyubiquitin chains of SDS3, which alters SDS3-associated HDAC activity [74]. However, deletion of HABMs in USP17 blocks the interaction between USP17 and SDS3 but retains the deubiquitinating activity of USP17 toward SDS3 [75].
Ras mutations, including H-Ras, N-Ras, and K-Ras, are common events that trigger carcinogenesis, and up to $30 \%$ of human cancers have these types of mutation [76]. It has been reported that USP17 inhibits the localization of $\mathrm{H}$-Ras and $\mathrm{N}$-Ras to the plasma membrane while not affecting the localization of K-Ras4b. In USP17-overexpressing cells, abundant N-Ras has been demonstrated to be located in the endoplasmic reticulum and Golgi. Abnormal localization of Ras induced by USP17 leads to downregulation of the MAPK, Mek/Erk, and PI3K/JNK signaling pathways, resulting in delayed growth of HeLa cells [77]. In addition, USP17 also deubiquitinates Ras converting enzyme 1 (RCE1), which is important for the activation of Ras. USP17 negatively regulates the activity of RCE1 by removing the K63-linked polyubiquitin chains conjugated to RCE1 [78]. These findings suggest that USP17 regulates differential Ras isoform signaling from different intracellular platforms, making it an important protein for further research related to potential cancer therapy.

ETS-like protein 1 (ELK-1) is a transcription factor that participates in Erk-induced cell proliferation [79]. The transcriptional activity of ELK- 1 is regulated by ubiquitination at lysine 35 . As a DUB, USP17 reverses the ubiquitination of ELK-1 and elevates the expression level of ELK-1-targeted genes, which is accompanied by activation of Cyclin D1. In contrast, the deletion of USP17 suppresses the expression level of ELK-1 target genes and inhibits cell proliferation [80]. This evidence suggests that USP17 is also involved in the regulation of cervical cancer cell proliferation through mediating the ELK-1 signaling pathway.

USP17 has a predominant role in regulating cell migration and cytoskeletal reorganization. In USP17-deficient cells, the protrusion decreases, the shape becomes round, and cytoskeletal polymerization is reduced, and the migration distance and migration rate significantly decrease. USP17 is induced by the stromal cell-derived factor-1 (SDF-1)/C-X-C motif chemokine ligand 12 (CXCL12) and IL-8/CXCL8 chemokines in cancer cells. However, the absence of USP17 hinders normal cytoskeletal rearrangement and chemokine-induced membrane localization of Rho GTPases, including CDC42, Rac, and RhoA, which are essential for cell motility [81]. These results suggest that USP17 is necessary for GTPase subcellular localization and cell motility, indicating that it may be a useful drug target for the treatment of cancer metastasis.

Ovarian cancer is the main cause of death of gynecological malignancies [82]. Immunohistochemistry analysis has shown that the expression level of DUB3 in ovarian cancer tissues is higher than that in normal ovarian tissues. The expression level of DUB3 is closely 
Table 1 The roles of USP17 in different types of cancers

\begin{tabular}{|c|c|c|c|}
\hline Cancer type & Targets & Functions & References \\
\hline \multirow[t]{2}{*}{ Central nervous system cancer } & Ras and Myc & Inhibits the carcinogenesis and proliferation of glioma & [35] \\
\hline & CDC25A & Negatively regulates cell cycle progression and proliferation of glioma & [36] \\
\hline \multirow[t]{2}{*}{ Head and neck cancer } & $\mathrm{BRD} 4 / \mathrm{EZH} 2$ & Promotes the proliferation of OSCC cells and inhibits apoptosis & [37] \\
\hline & Snail1 & Promotes the epithelial mesenchymal transition (EMT) & [38] \\
\hline \multirow[t]{6}{*}{ Thoracic cancer } & Cyclin A & $\begin{array}{l}\text { Drives cell cycle progression and promotes lung cancer cell prolifera- } \\
\text { tion }\end{array}$ & [41] \\
\hline & Transferrin and its receptor (TfR) & $\begin{array}{l}\text { Promotes the growth of EGFR mutation lung cancer cells and inhibits } \\
\text { apoptosis }\end{array}$ & [43] \\
\hline & TRAF2 and TRAF3 & $\begin{array}{l}\text { Upregulates the inflammation-related genes and stemness-related } \\
\text { genes in cancer cells }\end{array}$ & [45] \\
\hline & HAS2 & Triggers the progression of cancer & [47] \\
\hline & MMP3 and MMP9 & Promotes the tumorigenesis and growth & [48] \\
\hline & PI3KAkt & Contributes to cisplatin resistance in NSCLC cells & [49] \\
\hline \multirow[t]{6}{*}{ Breast cancer } & AEP & Promotes the intracellular stability and metastasis & {$[50]$} \\
\hline & SET8 & Increases the proliferation of breast cancer cells & [53] \\
\hline & Snail, Slug and Twist & $\begin{array}{l}\text { Promotes EMT and breast cancer metastasis; cancer stem cell-like } \\
\text { characteristics of breast cancer cells }\end{array}$ & {$[54,56]$} \\
\hline & CDC25A & Promotes cell cycle progression & [58] \\
\hline & Geminin & $\begin{array}{l}\text { Enhances genomic instability, DNA replication change, aneuploidy, } \\
\text { and cancer progression }\end{array}$ & [61] \\
\hline & MAPK signaling pathway & Decreases the cell viability & {$[62]$} \\
\hline \multirow[t]{3}{*}{ Gastrointestinal cancer } & $\mathrm{H} 2 \mathrm{AX}$ & Overcomes DNA double-strand break (DSB) & {$[66,68]$} \\
\hline & DEC1 & Recovers from G2 DNA damage checkpoint & [69] \\
\hline & Nrf2 & Leads to chemotherapy resistance & [70] \\
\hline \multirow[t]{2}{*}{ Genitourinary cancer } & $\mathrm{NF}-\mathrm{kB} / \mathrm{ROS}$ & $\begin{array}{l}\text { Promotes the proliferation, migration, and invasion of prostate cancer } \\
\text { cells and inhibits apoptosis }\end{array}$ & [72] \\
\hline & BRD4 & Leads to drug resistance of BET inhibitor & [73] \\
\hline \multirow[t]{5}{*}{ Gynecologic cancer } & SDS3 & $\begin{array}{l}\text { Induces apoptosis and inhibits cell proliferation in cervical adenocar- } \\
\text { cinoma cells }\end{array}$ & [74] \\
\hline & Ras & Decreases growth of Hela cells & [77] \\
\hline & ELK-1 & Promotes cell proliferation & {$[80]$} \\
\hline & Rho GTPases & Promotes cell migration and cytoskeleton reorganization & [81] \\
\hline & MCL1 & Regulates chemoresistance in ovarian cancer & {$[85]$} \\
\hline Bone and soft tissue sarcomas & Smad4 & $\begin{array}{l}\text { Enhances the migration and invasion of osteosarcoma cells through } \\
\text { activation of EMT }\end{array}$ & {$[86]$} \\
\hline
\end{tabular}

AEP asparaginyl endopeptidase, $A k t$ protein kinase $B, B E T$ bromodomain and extra-terminal domain, BRD4 bromodomain-containing protein 4, CDC25A cell division cycle 25A, DEC1 defective chorion-1, EGFR epidermal growth factor receptor, ELK-1 ETS-like protein 1, EZH2 enhancer of zeste homolog-2, H2AX H2A histone family member X, HAS2 hyaluronan synthase 2, MAPK mitogen-activated protein kinase, MCL1 myeloid cell leukemia sequence 1, MMP3 matrix metallopeptidase 3, OSCC oral squamous cell carcinoma, PI3K phosphoinositide-3-kinase, ROS reactive oxygen species, SDS3 suppressor of defective silencing 3, SET8 SET domain-containing protein 8, TRAFs tumor necrosis factor receptor associated factors

related to lymph node metastasis, advanced clinical stage, and poor prognosis. A previous in vitro study has shown that DUB3 silencing induces apoptosis and inhibits the proliferation of ovarian cancer cells by arresting the cell cycle in G0/G1 phase [83].

Chemotherapy is an important component of the treatment for ovarian cancer [84]. Myeloid cell leukemia sequence 1 (MCL1) plays a critical role in the regulation of chemoresistance in ovarian cancer. In ovarian cancer cells, DUB3 interacts with MCL1 to deubiquitinate and stabilize MCL1 through its 40th lysine at the N-terminus.
In addition, O6-methylguanine-DNA methyltransferase (MGMT) is a key activator of DUB3 transcription. The MGMT inhibitor, PaTrin-2, effectively inhibits DUB3 at the transcriptional level. Therefore, DUB3 suppression or PaTrin-2 treatment significantly induces apoptosis of ovarian cancer cells by downregulating MCL1. Furthermore, the expression of MGMT/DUB3 is activated by HDACis. A synergetic therapeutic effect has been shown with the combined use of HDACis and PaTrin-2. These results suggest that the MGMT-DUB3-MCL1 signaling 


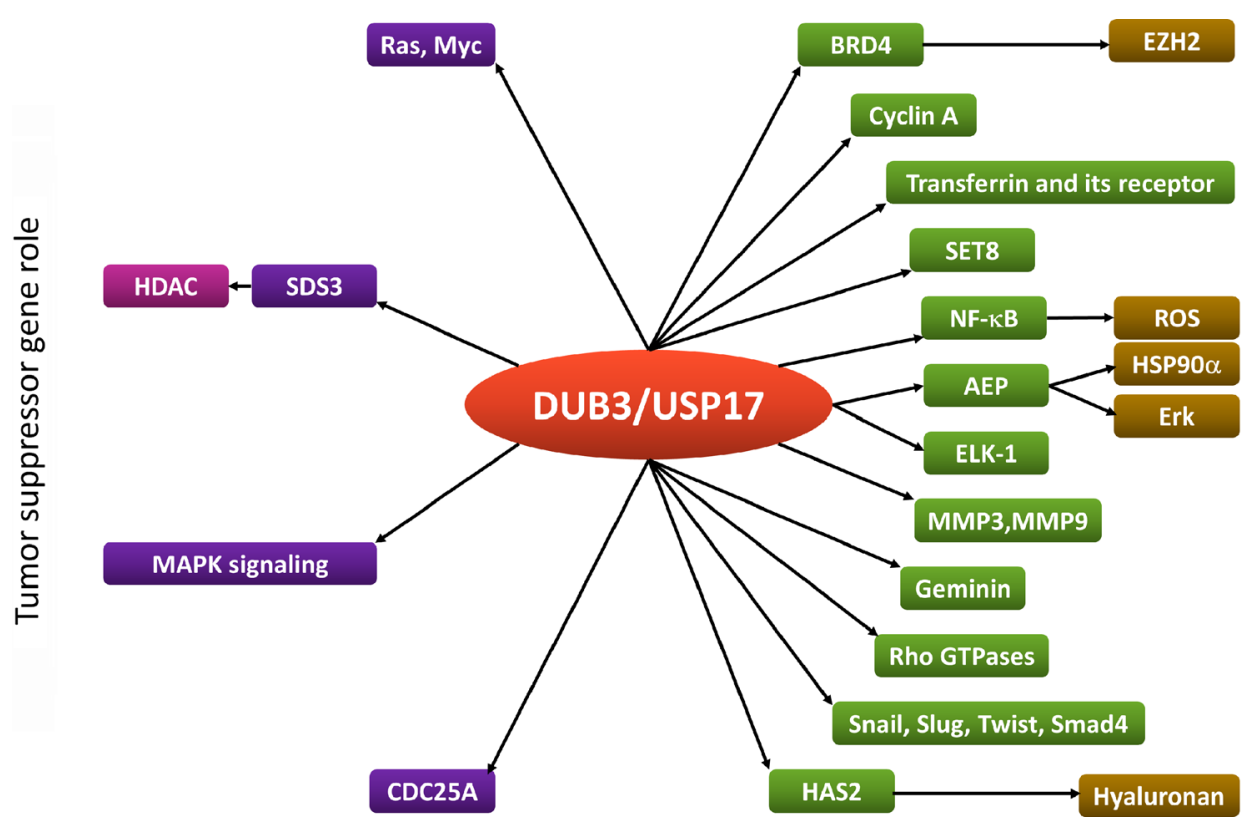

Fig. 3 The oncogenic and the tumor suppressor role of DUB3/USP17 in cancer and the pathways in which it participate

axis has a role in the regulation of chemoresistance in ovarian cancer [85].

\section{Bone and soft tissue sarcomas}

USP17 exerts an oncogenic role in osteosarcoma. The expression of USP17 is upregulated in osteosarcoma tissues, MG-63 cells, and U2OS cells. Functional experiments have shown that USP17 promotes the proliferation, migration, and invasion of osteosarcoma cells. USP17 interacts with Smad4 to stabilize Smad4 through its DUB activity and then promotes EMT to enhance the migration and invasion of osteosarcoma cells [86].

\section{Conclusions}

In the past decade, DUBs have become attractive targets for cancer treatment. As an important DUB, DUB3/ USP17 is involved in the regulation of most, if not all, cancer hallmarks, especially the signal transduction pathways that confer cell cycle progression, proliferation, apoptosis, and treatment resistance (Table 1). In this review, we summarized the oncogenic and the tumor suppressor role of DUB3/USP17 in cancer (Fig. 3). However, most of the recent studies have focused on the function of USP17 in regulating protein degradation and stability. In addition to participating in protein ubiquitination, the nonubiquitin function of USP17 also needs to be further studied in cancer. Furthermore, it is necessary to clarify how USP17 achieves specificity through substrate interactions in different tumor microenvironments because recent studies have shown that USP17 regulates the stability and nuclear function of IL-33 [87]. Finally, more data on USP17 may lead to the development of specific USP17 inhibitors/agonists for cancer treatment. Discovery of the role of USP17 in cancer may provide valuable information to prevent carcinogenesis and develop effective drugs.

\section{Abbreviations}

53BP1: P53-binding protein 1; AEP: Asparaginyl endopeptidase; AGO2: Argonaute 2; Akt: Protein kinase B; ATP: Adenosine triphosphate; BET: Bromodomain and extra-terminal domain; BLBC: Basal-like breast cancer; BRCA1: Breast cancer susceptibility gene 1; BRD4: Bromodomain-containing protein 4; CDC25A: Cell division cycle 25A; CDK4/6: Cyclin-dependent kinases 4 and 6; CDKls: Cyclin-dependent kinase inhibitors; Cdt1: Chromatin licensing and DNA replication factor 1; CME: Clathrin mediated endocytosis; CXCL12: C-X-C motif chemokine ligand 12; DDR: DNA damage response; DEC1: Defective chorion-1; DSB: DNA double-strand break; DUBs: Deubiquitinating enzymes; EGF: Epidermal growth factor; EMT: Epithelial mesenchymal transition; EZH2: Enhancer of zeste homolog-2; H2AX: H2A histone family member $X_{;}$HABMs: Hyaluronan (and RNA) binding motifs; HAS2: Hyaluronan synthase 2; HDAC: Histone deacetylase; HECT: Homologous to the E6-AP carboxyl terminus; HR: Homologous recombination; HSP90a: Heat shock protein 90a; IL-4: Interleukin-4; IRF5: Interferon regulatory factor 5; JAMMs: JAMM/MPN domainassociated metallopeptidases; MAPK: Mitogen-activated protein kinase; MCL1: Myeloid cell leukemia sequence 1; MCPIPS: Monocyte chemotactic protein-induced proteins; MGMT: 06-Methylguanine-DNA methyltransferase; MJDs: Machado-Joseph (Josephin) domain proteases; MMP3: Matrix metallopeptidase 3; NCOR2: Nuclear receptor corepressor 2; NHEJ: Non-homologous end joining; NIK: NF-kappaB-inducing kinase; NSCLC: Non-small cell lung cancer; OSCC: Oral squamous cell carcinoma; OTUs: Ovarian tumor proteases; PI3K: Phosphoinositide-3-kinase; PTEN: Phosphatase and tensin homologue; PTMs: Post-translational modifications; RBR: Ring between ring fingers; RCE1: Ras converting enzyme 1; RING: Really interesting new gene; ROS: Reactive oxygen species; SDF-1: Stromal cell-derived factor-1; SDS3: Suppressor of defective silencing 3: SET8: SET domain-containing protein 8; TfR:Transferrin and its receptor; Th17: T helper 17 cells; TKl: Tyrosine kinase inhibitor; TNBC: Triple negative breast cancer; TRAFs: Tumor necrosis factor receptor associated 
factors; UCHs: Ubiquitin COOH terminal hydrolases; USP 17: Ubiquitin-specific protease 17; ZEB1: Zinc finger E-box combined with homeobox 1.

\section{Acknowledgements}

We thank AJE for the language editing of this manuscript.

\section{Authors' contributions}

GFY, XZ, and YGS: conceptualization and writing. YYW: supervision, review, and editing. RZ: participation in study design and critical discussion. All authors read and approved the final manuscript.

\section{Funding}

This study was supported financially by the following foundations: National Natural Science Foundation of China (82060433), Ningxia Natural Science Foundation (2020AAC03419), and General Hospital of Ningxia Medical University providing a research grant for Yan-Yang Wang. Funding organizations have no role in the design or implementation of the study. They offered unrestricted grants.

\section{Availability of data and materials}

None.

\section{Declarations}

\section{Ethics approval and consent to participate}

Not applicable.

\section{Consent for publication}

Not applicable.

\section{Competing interests}

The authors declare no potential financial or non-financial conflicts of interest.

\section{Author details}

${ }^{1}$ Dept. of Ultrasound, General Hospital of Ningxia Medical University, Yinchuan 750004, Ningxia, China. ${ }^{2}$ School of Clinical Medicine, Ningxia Medical University, Yinchuan 750004, Ningxia, China. ${ }^{3}$ Graduate School, Ningxia Medical University, Yinchuan 750004, Ningxia, China. ${ }^{4}$ Dept. of Radiation Oncology, General Hospital of Ningxia Medical University, Yinchuan 750004, Ningxia, China. ${ }^{5}$ Cancer Institute, Ningxia Medical University, Yinchuan 750004, Ningxia, China.

Received: 25 April 2021 Accepted: 18 August 2021

Published online: 28 August 2021

\section{References}

1. Hicke L, Dunn R. Regulation of membrane protein transport by ubiquitin and ubiquitin-binding proteins. Annu Rev Cell Dev Biol. 2003;19:141-72.

2. Komander D, Rape M. The ubiquitin code. Annu Rev Biochem. 2012;81:203-29.

3. Ciechanover A, Kwon YT. Degradation of misfolded proteins in neurodegenerative diseases: therapeutic targets and strategies. Exp Mol Med. 2015;47:e147.

4. Shi D, Grossman SR. Ubiquitin becomes ubiquitous in cancer: emerging roles of ubiquitin ligases and deubiquitinases in tumorigenesis and as therapeutic targets. Cancer Biol Ther. 2010;10(8):737-47.

5. Pickart CM, Eddins MJ. Ubiquitin: structures, functions, mechanisms. Biochim Biophys Acta. 2004;1695(1-3):55-72.

6. Heideker J, Wertz IE. DUBS, the regulation of cell identity and disease. Biochem J. 2015:467(1):191.

7. Haq S, Ramakrishna S. Deubiquitylation of deubiquitylases. Open Biol. 2017;7(6):170016

8. Komander D, Clague MJ, Urbe S. Breaking the chains: structure and function of the deubiquitinases. Nat Rev Mol Cell Biol. 2009;10(8):550-63.

9. Young MJ, Hsu KC, Lin TE, Chang WC, Hung JJ. The role of ubiquitinspecific peptidases in cancer progression. J Biomed Sci. 2019;26(1):42.
10. Burrows JF, McGrattan MJ, Rascle A, Humbert M, Baek KH, Johnston JA. DUB-3, a cytokine-inducible deubiquitinating enzyme that blocks proliferation. J Biol Chem. 2004;279(14):13993-4000.

11. Han L, Yang J, Wang X, Wu Q, Yin S, Li Z, et al. The E3 deubiquitinase USP17 is a positive regulator of retinoic acid-related orphan nuclear receptor gammat (RORgammat) in Th17 cells. J Biol Chem. 2014;289(37):25546-55.

12. Pickart CM. Mechanisms underlying ubiquitination. Annu Rev Biochem. 2001;70:503-33.

13. Ciechanover $A$. The ubiquitin-proteasome pathway: on protein death and cell life. EMBO J. 1998;17(24):7151-60.

14. Koegl M, Hoppe T, Schlenker S, Ulrich HD, Mayer TU, Jentsch S. A novel ubiquitination factor, E4, is involved in multiubiquitin chain assembly. Cell. 1999:96(5):635-44.

15. Metzger MB, Hristova VA, Weissman AM. HECT and RING finger families of E3 ubiquitin ligases at a glance. J Cell Sci. 2012;125(Pt 3):531-7.

16. Zheng N, Wang P, Jeffrey PD, Pavletich NP. Structure of a c-Cbl-UbcH7 complex: RING domain function in ubiquitin-protein ligases. Cell. 2000;102(4):533-9.

17. Plechanovova A, Jaffray EG, Tatham MH, Naismith JH, Hay RT. Structure of a RING E3 ligase and ubiquitin-loaded E2 primed for catalysis. Nature. 2012:489(7414):115-20.

18. Kim HC, Huibregtse JM. Polyubiquitination by HECT E3s and the determinants of chain type specificity. Mol Cell Biol. 2009;29(12):3307-18.

19. Spratt DE, Walden H, Shaw GS. RBR E3 ubiquitin ligases: new structures, new insights, new questions. Biochem J. 2014;458(3):421-37.

20. Lechtenberg BC, Rajput A, Sanishvili R, Dobaczewska MK, Ware CF, Mace PD, et al. Structure of a HOIP/E2 ubiquitin complex reveals RBR E3 ligase mechanism and regulation. Nature. 2016;529(7587):546-50.

21. Komander $\mathrm{D}$. The emerging complexity of protein ubiquitination. Biochem Soc Trans. 2009:37(Pt 5):937-53.

22. Hoeller D, Dikic I. Regulation of ubiquitin receptors by coupled monoubiquitination. Subcell Biochem. 2010;54:31-40.

23. Hicke L. Protein regulation by monoubiquitin. Nat Rev Mol Cell Biol. 2001;2(3):195-201.

24. Amerik AY, Hochstrasser M. Mechanism and function of deubiquitinating enzymes. Biochim Biophys Acta. 2004;1695(1-3):189-207.

25. Clague MJ, Barsukov I, Coulson JM, Liu H, Rigden DJ, Urbe S. Deubiquitylases from genes to organism. Physiol Rev. 2013;93(3):1289-315.

26. Reyes-Turcu FE, Ventii KH, Wilkinson KD. Regulation and cellular roles of ubiquitin-specific deubiquitinating enzymes. Annu Rev Biochem. 2009:78:363-97.

27. Nijman SM, Luna-Vargas MP, Velds A, Brummelkamp TR, Dirac AM, Sixma TK, et al. A genomic and functional inventory of deubiquitinating enzymes. Cell. 2005;123(5):773-86.

28. Sippl W, Collura V, Colland F. Ubiquitin-specific proteases as cancer drug targets. Future Oncol. 2011;7(5):619-32.

29. Hussain S, Zhang Y, Galardy PJ. DUBs and cancer: the role of deubiquitinating enzymes as oncogenes, non-oncogenes and tumor suppressors. Cell Cycle. 2009;8(11):1688-97.

30. Ducker C, Shaw PE. USP17-mediated de-ubiquitination and cancer: clients cluster around the cell cycle. Int J Biochem Cell Biol. 2021;130:105886

31. Burrows JF, Scott CJ, Johnston JA. The DUB/USP17 deubiquitinating enzymes: a gene family within a tandemly repeated sequence, is also embedded within the copy number variable beta-defensin cluster. BMC Genom. 2010:11:250.

32. Hjortland NM, Mesecar AD. Steady-state kinetic studies reveal that the anti-cancer target ubiquitin-specific protease 17 (USP17) is a highly efficient deubiquitinating enzyme. Arch Biochem Biophys. 2016;612:35-45.

33. Shin JM, Yoo KJ, Kim MS, Kim D, Baek KH. Hyaluronan- and RNA-binding deubiquitinating enzymes of USP17 family members associated with cell viability. BMC Genom. 2006;7:292.

34. Zeng T, Cui D, Gao L. Glioma: an overview of current classifications, characteristics, molecular biology and target therapies. Front Biosci. 2015;20:1104-15

35. Hu M, Chen H, Han C, Lan J, Xu Y, Li C, et al. Expression and functional implications of USP17 in glioma. Neurosci Lett. 2016;616:125-31.

36. Forte S, Pagliuca A, Maniscalchi ET, Gulino R, Calabrese G, Ricci-Vitiani L, et al. Gene expression analysis of PTEN positive glioblastoma stem cells 
identifies DUB3 and Wee1 modulation in a cell differentiation model. PLOS ONE. 2013;8(12):e81432.

37. Luo F, Zhou Z, Cai J, Du W. DUB3 facilitates growth and inhibits apoptosis through enhancing expression of EZH2 in oral squamous cell carcinoma. Onco Targets Ther. 2020;13:1447-60.

38. Feng L, Zhang J, Sun M, Qiu F, Chen W, Qiu W. Tumor suppressor LINC02487 inhibits oral squamous cell carcinoma cell migration and invasion through the USP17-SNAI1 axis. Front Oncol. 2020;10:559808.

39. McFarlane C, McFarlane S, Paul I, Arthur K, Scheaff M, Kerr K, et al. The deubiquitinating enzyme USP17 is associated with non-smal cell lung cancer (NSCLC) recurrence and metastasis. Oncotarget. 2013;4(10):1836-43

40. Darling S, Fielding AB, Sabat-Pospiech D, Prior IA, Coulson JM. Regulation of the cell cycle and centrosome biology by deubiquitylases. Biochem Soc Trans. 2017;45(5):1125-36.

41. Hu B, Deng T, Ma H, Liu Y, Feng P, Wei D, et al. Deubiquitinase DUB3 regulates cell cycle progression via stabilizing cyclin A for proliferation of non-small cell lung cancer cells. Cells. 2019;8(4):297.

42. Jaworski J, de la Vega M, Fletcher SJ, McFarlane C, Greene MK, Smyth AW, et al. USP17 is required for clathrin mediated endocytosis of epidermal growth factor receptor. Oncotarget. 2014;5(16):6964-75.

43. McCann AP, Smyth P, Cogo F, McDaid WJ, Jiang L, Lin J, et al. USP17 is required for trafficking and oncogenic signaling of mutant EGFR in NSCLC cells. Cell Commun Signal. 2018;16(1):77.

44. Conway EM, Pikor LA, Kung SH, Hamilton MJ, Lam S, Lam WL, et al. Macrophages, inflammation, and lung cancer. Am J Respir Crit Care Med. 2016;193(2):116-30

45. Lu CH, Yeh DW, Lai CY, Liu YL, Huang LR, Lee AY, et al. USP17 mediates macrophage-promoted inflammation and stemness in lung cancer cells by regulating TRAF2/TRAF3 complex formation. Oncogene. 2018:37(49):6327-40.

46. Toole BP. Hyaluronan: from extracellular glue to pericellular cue. Nat Rev Cancer. 2004;4(7):528-39.

47. Mehic M, de Sa VK, Hebestreit S, Heldin CH, Heldin P. The deubiquitinating enzymes USP4 and USP17 target hyaluronan synthase 2 and differentially affect its function. Oncogenesis. 2017;6(6):e348.

48. Zhang S, Yuan J, Zheng R. Suppression of ubiquitin-specific peptidase 17 (USP17) inhibits tumorigenesis and invasion in non-small cell lung cancer cells. Oncol Res. 2016;24(4):263-9.

49. Zhang $\mathrm{S}, \mathrm{XuZ}$ Z, Yuan J, Chen H. Ubiquitin-specific peptidase 17 promotes cisplatin resistance via PI3K/AKT activation in non-small cell lung cancer. Oncol Lett. 2020;20(1):67-74.

50. Lin Y, Qiu Y, Xu C, Liu Q, Peng B, Kaufmann GF, et al. Functional role of asparaginyl endopeptidase ubiquitination by TRAF6 in tumor invasion and metastasis. J Natl Cancer Inst. 2014;106(4):dju012.

51. Chen X, Wang C, Liao K, Zhou S, Cao L, Chen J, et al. USP17 suppresses tumorigenesis and tumor growth through deubiquitinating AEP. Int J Biol Sci. 2019;15(4):738-48.

52. Couture JF, Collazo E, Brunzelle JS, Trievel RC. Structural and functional analysis of SET8, a histone H4 Lys-20 methyltransferase. Genes Dev. 2005;19(12):1455-65.

53. Fukuura K, Inoue Y, Miyajima C, Watanabe S, Tokugawa M, Morishita D, et al. The ubiquitin-specific protease USP17 prevents cellular senescence by stabilizing the methyltransferase SET8 and transcriptionally repressing p21. J Biol Chem. 2019;294(44):16429-39.

54. Liu T, Yu J, Deng M, Yin Y, Zhang H, Luo K, et al. CDK4/6-dependent activation of DUB3 regulates cancer metastasis through SNAIL1. Nat Commun. 2017;8:13923.

55. Wu Y, Wang Y, Lin Y, Liu Y, Wang Y, Jia J, et al. Dub3 inhibition suppresses breast cancer invasion and metastasis by promoting Snail1 degradation. Nat Commun. 2017;8:14228

56. Lin Y, Wang Y, Shi Q, Yu Q, Liu C, Feng J, et al. Stabilization of the transcription factors slug and twist by the deubiquitinase dub3 is a key requirement for tumor metastasis. Oncotarget. 2017;8(43):75127-40.

57. Kristjansdottir K, Rudolph J. Cdc25 phosphatases and cancer. Chem Biol. 2004;11(8):1043-51.

58. Pereg Y, Liu BY, O'Rourke KM, Sagolla M, Dey A, Komuves L, et al. Ubiquitin hydrolase Dub3 promotes oncogenic transformation by stabilizing Cdc25A. Nat Cell Biol. 2010;12(4):400-6.

59. McFarlane C, Kelvin AA, de la Vega M, Govender U, Scott CJ, Burrows JF, et al. The deubiquitinating enzyme USP17 is highly expressed in tumor biopsies, is cell cycle regulated, and is required for G1-S progression. Cancer Res. 2010;70(8):3329-39.

60. McGarry TJ, Kirschner MW. Geminin, an inhibitor of DNA replication, is degraded during mitosis. Cell. 1998;93(6):1043-53.

61. Hernandez-Perez S, Cabrera E, Salido E, Lim M, Reid L, Lakhani SR, et al. DUB3 and USP7 de-ubiquitinating enzymes control replication inhibitor Geminin: molecular characterization and associations with breast cancer. Oncogene. 2017;36(33):4802-9.

62. Borbely G, Haldosen LA, Dahlman-Wright K, Zhao C. Induction of USP17 by combining BET and HDAC inhibitors in breast cancer cells. Oncotarget. 2015;6(32):33623-35.

63. Brinker AM, Ma J, Lipsky PE, Raskin I. Medicinal chemistry and pharmacology of genus Tripterygium (Celastraceae). Phytochemistry. 2007;68(6):732-66.

64. Cheng S, Zhang Z, Hu C, Xing N, Xia Y, Pang B. Pristimerin suppressed breast cancer progression via miR-542-5p/DUB3 axis. Onco Targets Ther. 2020;13:6651-60.

65. Zhang P, Sun Y, Ma L. ZEB1: at the crossroads of epithelial-mesenchymal transition, metastasis and therapy resistance. Cell Cycle. 2015;14(4):481-7.

66. Wang M, He SF, Liu LL, Sun XX, Yang F, Ge Q, et al. Potential role of ZEB1 as a DNA repair regulator in colorectal cancer cells revealed by cancerassociated promoter profiling. Oncol Rep. 2017;38(4):1941-8.

67. Rogakou EP, Pilch DR, Orr AH, Ivanova VS, Bonner WM. DNA doublestranded breaks induce histone H2AX phosphorylation on serine 139. J Biol Chem. 1998;273(10):5858-68.

68. Delgado-Diaz MR, Martin Y, Berg A, Freire R, Smits VA. Dub3 controls DNA damage signalling by direct deubiquitination of H2AX. Mol Oncol. 2014;8(5):884-93.

69. Kim J, D'Annibale S, Magliozzi R, Low TY, Jansen P, Shaltiel IA, et al. USP17- and SCFbetaTrCP-regulated degradation of DEC1 controls the DNA damage response. Mol Cell Biol. 2014;34(22):4177-85.

70. Zhang Q, Zhang ZY, Du H, Li SZ, Tu R, Jia YF, et al. DUB3 deubiquitinates and stabilizes NRF2 in chemotherapy resistance of colorectal cancer. Cell Death Differ. 2019;26(11):2300-13.

71. Gundem G, Van Loo P, Kremeyer B, Alexandrov LB, Tubio JMC, Papaem-

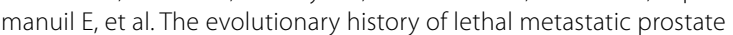
cancer. Nature. 2015;520(7547):353-7.

72. Baohai $X$, Shi F, Yongqi F. Inhibition of ubiquitin specific protease 17 restrains prostate cancer proliferation by regulation of epithelial-tomesenchymal transition (EMT) via ROS production. Biomed Pharmacother 2019:118:108946.

73. Jin X, Yan Y, Wang D, Ding D, Ma T, Ye Z, et al. DUB3 promotes BET inhibitor resistance and cancer progression by deubiquitinating BRD4. Mol Cell. 2018;71(4):592-605.e594.

74. Ramakrishna S, Suresh B, Lee EJ, Lee HJ, Ahn WS, Baek KH. Lys-63-specific deubiquitination of SDS3 by USP17 regulates HDAC activity. J Biol Chem. 2011;286(12):10505-14.

75. Ramakrishna S, Suresh B, Bae SM, Ahn WS, Lim KH, Baek KH. Hyaluronan binding motifs of USP17 and SDS3 exhibit anti-tumor activity. PLOS ONE. 2012;7(5):e37772.

76. Malumbres M, Barbacid M. To cycle or not to cycle: a critical decision in cancer. Nat Rev Cancer. 2001;1(3):222-31.

77. de la Vega M, Burrows JF, McFarlane C, Govender U, Scott CJ, Johnston $J A$. The deubiquitinating enzyme USP17 blocks N-Ras membrane trafficking and activation but leaves K-Ras unaffected. J Biol Chem. 2010;285(16):12028-36.

78. Burrows JF, Kelvin AA, McFarlane C, Burden RE, McGrattan MJ, De la Vega $\mathrm{M}$, et al. USP17 regulates Ras activation and cell proliferation by blocking RCE1 activity. J Biol Chem. 2009;284(14):9587-95.

79. Boros J, Donaldson IJ, O'Donnell A, Odrowaz ZA, Zeef L, Lupien M, et al. Elucidation of the ELK1 target gene network reveals a role in the coordinate regulation of core components of the gene regulation machinery. Genome Res. 2009;19(11):1963-73.

80. Ducker C, Chow LKY, Saxton J, Handwerger J, McGregor A, Strahl T, et al. De-ubiquitination of ELK-1 by USP17 potentiates mitogenic gene expression and cell proliferation. Nucleic Acids Res. 2019;47(9):4495-508.

81. de la Vega M, Kelvin AA, Dunican DJ, MCFarlane C, Burrows JF, Jaworski J, et al. The deubiquitinating enzyme USP17 is essential for GTPase subcellular localization and cell motility. Nat Commun. 2011;2:259. 
82. Chi DS, Eisenhauer EL, Zivanovic O, Sonoda Y, Abu-Rustum NR, Levine DA, et al. Improved progression-free and overall survival in advanced ovarian cancer as a result of a change in surgical paradigm. Gynecol Oncol. 2009;114(1):26-31.

83. Zhou B, Shu B, Xi T, Su N, Liu J. Dub3 expression correlates with tumor progression and poor prognosis in human epithelial ovarian cancer. Biomed Pharmacother. 2015;70:84-9.

84. Bast RC Jr, Hennessy B, Mills GB. The biology of ovarian cancer: new opportunities for translation. Nat Rev Cancer. 2009;9(6):415-28.

85. Wu X, Luo Q, Zhao P, Chang W, Wang Y, Shu T, et al. MGMT-activated DUB3 stabilizes MCL1 and drives chemoresistance in ovarian cancer. Proc Natl Acad Sci USA. 2019;116(8):2961-6.
86. Song C, Liu W, Li J. USP17 is upregulated in osteosarcoma and promotes cell proliferation, metastasis, and epithelial-mesenchymal transition through stabilizing SMAD4. Tumour Biol. 2017;39(7):1010428317717138.

87. Ni Y, Tao L, Chen C, Song H, Li Z, Gao Y, et al. The deubiquitinase USP17 regulates the stability and nuclear function of IL-33. Int J Mol Sci. 2015;16(11):27956-66.

\section{Publisher's Note}

Springer Nature remains neutral with regard to jurisdictional claims in published maps and institutional affiliations.
Ready to submit your research? Choose BMC and benefit from:

- fast, convenient online submission

- thorough peer review by experienced researchers in your field

- rapid publication on acceptance

- support for research data, including large and complex data types

- gold Open Access which fosters wider collaboration and increased citations

- maximum visibility for your research: over 100M website views per year

At BMC, research is always in progress.

Learn more biomedcentral.com/submissions 